\title{
Limitada publicación de tesis de pregrado en una facultad de medicina de Lima, Perú, 2000-2009
}

Limited scientific publication of pre-graduate theses in a faculty of medicine in Lima,

Peru, 2000-2009

\author{
Álvaro Taype-Rondán ${ }^{1, a}$, Claudia Carbajal-Castro ${ }^{1, a}$, Giuliana Arrunategui-Salas, , \\ Javier Chambi-Torres ${ }^{1, a}$ \\ 'Sociedad Cientifica de Estudiantes de Medicina, Universidad de San Martin de Porres (SOCIEM-USMP), Lima, Perú.
}

${ }^{\text {a }}$ Estudiante de Medicina Humana.

\begin{abstract}
Resumen
Introducción: Los autores de tesis de pregrado deben buscar publicar sus investigaciones en revistas cientificas, para respaldar su calidad y garantizar su difusión en el ámbito académico-científico. Objetivo: Determinar la publicación de las tesis de pregrado de la Facultad de Medicina Humana de la Universidad de San Martín de Porres aprobadas en el periodo 2000-2009. Diseño: observacional, descriptivo. Materiales: Tesis de pregrado aprobadas en el periodo 2000-2009. Intervenciones: Se solicitó las tesis en la biblioteca de dicha universidad. Se realizó una búsqueda de cada tesis en Google Académico para determinar si fueron publicadas. Además, se buscó la producción científica de los asesores y los jurados de cada tesis. Estos datos fueron tabulados en Microsoft Excel 2010 y presentados en forma descriptiva. Principales medidas de los resultados: Publicación de las tesis, producción científica de asesores y jurados. Resultados: Durante el periodo 2000-2009, egresaron 2667 estudiantes y se aprobó 74 tesis de pregrado, de las cuales solo dos fueron publicadas. Se encontró que 21 de 67 asesores $(31,3 \%)$ y 24 de 73 jurados $(32,9 \%)$ tuvieron algún artículo original publicado. Conclusiones: Se encuentra una proporción baja de tesis publicadas en la facultad estudiada.
\end{abstract}

Palabras clave: Tesis académicas, bibliometría, estudiantes de medicina, escuelas médicas.

Abstract

Background: Authors of pre-graduate theses must try to publish them in scientific journals in order to support their quality and diffusion in the scientific-academic environment. Objectives: To determine Universidad de San Martin de Porres' School of Medicine approved pregraduate thesis publication in the period 2000-2009. Design: Observational, descriptive. Materials: Pre-graduate theses approved during 2000-2009. Interventions: Permission was obtained to access theses at the University library. A search for separate theses was conducted utilizing Google Scholar to find dates of publication. Personal scientific production of both theses consultants and jurors was also researched. Results were tabulated in Microsoft Excel 2010 and presented descriptively. Main outcome measures: Theses publication, scientific production of tutors and juries. Results: During 2000-2009, 2667 students graduated, 74 theses were approved, and only two were published. Twenty-one out of 67 consultants (31.3\%) and 24 out of 73 jurors (32.9\%) had at least one original article published. Conclusions: Low proportion of thesis publication was found at the faculty of Medicine studied.

Key words: Academic dissertations, bibliometrics, students/medical, schools/medical.

An Fac med. 2012;73(2):153-7

\section{INTRODUCCIÓN}

La investigación científica en medicina es una herramienta importante que permite mejorar la salud de la población. Resulta necesario motivar a los médicos a realizar y publicar trabajos de investigación desde el pregrado, pues se sabe que los estudiantes de pregrado que realizan trabajos de investigación tienden a seguir investigando una vez graduados ${ }^{(1,2)}$. En el Perú, la publicación científica estudiantil crece cada año, aunque la mayor parte de estas publicaciones son realizadas por estudiantes de dos facultades de medicina de nuestro país ${ }^{(3)}$.
Las tesis de pregrado son trabajos de investigación que los estudiantes pueden realizar para optar por el título de médico cirujano. Sin embargo, en el Perú hay otras maneras de obtener dicho título, pues rige desde el año 1991 el Decreto Legislativo № 739, que permite que los títulos universitarios sean entregados bajo la modalidad que las universidades consideren pertinente ${ }^{(4)}$.

El fin de una investigación es generar un conocimiento que pueda ser útil ya sea para la toma de decisiones o para la formulación de nuevos trabajos de investigación; además, es necesario que estos trabajos sean publicados en revistas científicas indizadas, para res- paldar su calidad y permitir su difusión entre la comunidad médica y científica. Estas revistas evalúan con exigencia la calidad de los artículos, sometiéndolos para ello a revisiones por expertos en el tema ${ }^{(5)}$, lo que respalda su calidad, para luego garantizar su difusión en el ámbito académico-científico.

En la actualidad existen aplicaciones que ayudan a promover la publicación y difusión de tesis electrónicas, como por ejemplo la plataforma Cybertesis ${ }^{(6)}$; sin embargo, dichas tesis pudieran no tener la rigurosidad de una revisión por pares y ser desestimadas para otras investigaciones. 
Dada la importancia de las tesis de pregrado en la educación médica, se ha analizado la publicación de tesis en dos facultades de medicina peruanas: la Universidad Nacional Mayor de San Marcos (UNMSM) (7) y la Universidad Peruana Cayetano Heredia (UPCH) ${ }^{(8)}$. Dichos estudios no permiten analizar toda la publicación de las tesis de pregrado en el Perú, a pesar que estas universidades tienen la mayor producción científica del país ${ }^{(7,8)}$. Entonces, es necesario analizar dicho fenómeno también en otras facultades de medicina.

Es por ello que el presente trabajo tiene como objetivo cuantificar la publicación de las tesis de pregrado de la Facultad de Medicina Humana de la Universidad de San Martín de Porres (FMH-USMP) aprobadas en el periodo 2000-2009.

\section{MÉTODOS}

Se realizó un estudio observacional, descriptivo, analizando todas las tesis de pregrado de la FMH-USMP aprobadas durante al período 2000-2009, las cuales fueron solicitadas en la biblioteca de dicha facultad. Además, se solicitó en la oficina de secretaría de la FMH-USMP información sobre la cantidad de egresados por año, durante el periodo 2000-2009.

En una primera etapa se revisó cada tesis, describiendo su año de registro, título, número de autores estudiantes, nombres de los autores estudiantes, nombres de los asesores, nombres de los jurados, diseño de estudio, lugar donde fue realizada, número de referencias bibliográficas, referencias con una antigüedad menor de 5 años y referencias que no mencionan el año. Dichas variables fueron tabuladas en una base de datos elaborada en el programa Microsoft Excel 2010. Cada variable fue tabulada por dos investigadores diferentes. Luego se comparó ambas tabulaciones y las incongruencias fueron revisadas por todos los autores. El diseño de las tesis fue obtenido de la sección de materiales y métodos; se buscó los nombres de los asesores y de los miembros de jurados en las carátulas y las secciones de materiales y métodos, agradecimientos y anexos de las tesis.

En una segunda etapa, se utilizó el buscador Google Académico para determinar la publicación de la tesis, el número de citas de las tesis publicadas y la producción científica de asesores y de jurados.

Para verificar la publicación de las tesis, se hizo una búsqueda bibliográfica en Google Académico, con una combinación de tres términos de búsqueda (lugar de estudio, población de estudio y resultado principal) y el primer apellido del autor estudiante, tanto en español como en inglés, durante enero del año 2012. Esta estrategia de búsqueda ha sido utilizada con anterioridad en otros estudios ${ }^{(9,10)}$. El número de citas para cada tesis publicada fue también obtenido de Google Académico.

Durante enero del año 2012, se realizó búsquedas en Google Académico usando una combinación de las palabras clave "Lima" y "Perú", con varias combinaciones de los nombres y apellidos de cada miembro del jurado y cada asesor, para determinar si estos habían sido autores de algún artículo publicado en una revista científica, autores de algún artículo original publicado en una revista científica y autores principales de algún artículo original en una revista científica. El buscador Google Académico fue elegido debido a que incluye los artículos publicados en una gran cantidad de revistas científicas; teóricamente, contiene a todas las revistas científicas electrónicas existentes, a nivel internacional ${ }^{(11)}$.

Por último, se efectuó un análisis descriptivo de los resultados en el programa Microsoft Excel 2010, para presentarlos usando frecuencias y porcentajes.

\section{RESULTADOS}

De los 2667 estudiantes que se graduaron en el periodo 2000-2009 de la FMH-USMP, solo 75 lo hicieron bajo la modalidad de tesis. Se identificó 74 tesis en los registros de la biblioteca de la FMH-USMP, de las cuales 73 tuvieron un solo autor y una tuvo dos autores. La distribución anual de graduación por tesis se observa en la tabla 1.

Sobre el diseño de investigación según lo mencionado en la sección de materiales y métodos de las tesis, se encontró que 45 tesis $(60,8 \%)$ fueron descriptivas y $11(14,9 \%)$ analíticas. Asimismo, 43 (58,1\%) fueron retros-

Tabla 1. Número de egresados y tesis aprobadas en la FMH-USMP durante el período 2000-2009.

\begin{tabular}{ccc} 
Año & Egresados & $\begin{array}{c}\text { Estudiantes que se } \\
\text { graduaron por tesis }\end{array}$ \\
2000 & 195 & $2(1,0 \%)$ \\
2001 & 232 & $5(2,2 \%)$ \\
2002 & 208 & $4(1,9 \%)$ \\
2003 & 356 & $9(2,5 \%)^{*}$ \\
2004 & 266 & $12(4,5 \%)$ \\
2005 & 281 & $25(8,9 \%)$ \\
2006 & 283 & $4(1,4 \%)$ \\
2007 & 224 & $10(4,5 \%)$ \\
2008 & 294 & $2(0,7 \%)$ \\
2009 & 328 & $2(0,6 \%)$ \\
\hline Total & 2667 & $75(2,8 \%)$ \\
\hline
\end{tabular}

\footnotetext{
* Una tesis del año 2003 tuvo a dos estudiantes como autores.
} 
pectivas y $8(10,8 \%)$ prospectivas; 24 $(32,4 \%)$ fueron trasversales y $4(5,4 \%)$ longitudinales.

De las tesis revisadas, 59 (79,7\%) fueron realizadas en hospitales o institutos de Lima y Callao y 8 (10,8\%) en el interior del país. Cabe resaltar que 4 tesis $(5,4 \%)$ fueron ejecutadas en los laboratorios de la FMH-USMP, como se detalla en la tabla 2.

En cuanto a las referencias bibliográficas, se analizó un total de 3880 referencias correspondientes a las 74 tesis, lo cual da un promedio de 52,4 referencias por tesis, con un mínimo de $9 \mathrm{y}$ un máximo de 201. En 102 referencias $(2,6 \%)$ no se mencionó el año y 1528 referencias $(39,4 \%)$ tuvieron menos de 5 años de antigüedad, tomando como referencia el año de sustentación de la tesis.

Al buscar cada tesis en Google Académico, se halló que dos tesis $(2,7 \%)$ fueron publicadas. Una de estas fue aprobada por la FMH-USMP en el año 2004 y publicada en 2005, como artículo original en la Revista de Gastroenterología del Perú, y a la fecha ha sido citada por 11 artículos; la otra fue aprobada en el año 2006 y publicada en 2009 como una short communication en la revista Genetics and molecular Bio- logy, y a la fecha no ha sido citada por artículos científicos. La primera tesis tuvo un asesor y la segunda dos asesores. Los tres asesores involucrados contaron con otras publicaciones originales en Google Académico. En ambas tesis no se menciona a los jurados.

Los asesores fueron mencionados en 61 tesis $(82,4 \%)$, de las cuales 43 (70,5\%) tuvieron un asesor, 16 (26,2\%) dos asesores, y dos (3,3\%) tres asesores. Sin embargo, dado que algunas tesis compartieron asesores, se contabilizó en total 67 asesores diferentes. Para analizar su producción científica, se excluyó a uno de los asesores, pues solo se mencionaba un nombre y un apellido del mismo, lo cual imposibilitó la búsqueda bibliográfica. De los 66 asesores restantes, 28 (41,8\%) tuvieron al menos un artículo publicado, 21 (31,3\%) al menos un artículo original publicado y $16(23,9 \%)$ fueron autores principales de al menos un artículo original publicado en una revista científica indizada.

Los jurados evaluadores fueron mencionados en 44 tesis $(59,5 \%)$, cada una de las cuales contó con tres miembros. Se encontró en total 73 miembros diferentes. De estos, 37 (49,3\%) tuvieron al menos una publicación, 24 (32,9\%) han publicado al menos un artículo original y $10(13,7 \%)$ han sido autores

Tabla 2. Instituciones en las cuales fueron realizadas las tesis de pregrado de la FMHUSMP en el periodo 2000-2009 $(n=74)$.

\begin{tabular}{lc} 
Instituciones & Tesis \\
Hospitales & $56(7)^{*}$ \\
Lima y Callao & $51(68,9)$ \\
Otras provincias & $5(6,8)$ \\
Institutos & $11(14,9)$ \\
Lima y Callao & $8(10,8)$ \\
Otras provincias & $3(4,1)$ \\
Laboratorios de investigación & $4(5,4)$ \\
Centro de Genética y Biología Molecular de la FMH-USMP & $3(4,1)$ \\
Centro de medicina tradicional y andina de la FMH-USMP & $1(1,3)$ \\
Colegios y guarderías de Lima & $2(2,7)$ \\
No especifica & $2(2,7)$ \\
\hline
\end{tabular}

*Las tesis suman más de $100 \%$, debido a que una tesis fue realizada en dos lugares.

principales de al menos un artículo original publicado en una revista científica indizada.

\section{DISCUSIÓN}

Durante el periodo 2000-2009, en la FMH-USMP se aprobó en promedio 7,4 tesis por año. En la Facultad de Medicina Humana de la UNMSM se aprobó 8,5 tesis por año, durante el periodo 1998-2008 (7), cifra que no podemos comparar con la nuestra, por no contar con información sobre el número de graduados de la UNMSM, ni el número de autores estudiantes por cada tesis. También se conoce que en la Facultad de Medicina Humana de la UPCH ${ }^{(8)}$ se aprobó 120,5 tesis por año en el periodo 2000-2003; sin embargo, es posible que en ese caso la tesis haya sido la única modalidad de titulación, por lo que tampoco se pueden establecer comparaciones con nuestros resultados.

En la FMH-USMP, es notorio que la mayoría de los estudiantes prefiere dar un examen de grado a sustentar una tesis. Esta problemática ya ha sido abordada por publicaciones anteriores, en las cuales se describe que los estudiantes carecen de tiempo (suelen realizar la tesis durante su internado) ${ }^{(12)}$, no cuentan con financiamiento, no se perciben capacitados en metodología de investigación ${ }^{(13)}$ ni redacción científica, perciben que no se valora el trabajo realizado, que no existe una cultura de publicación, que los trámites son engorrosos y que no tienen apoyo por parte de su universidad ${ }^{(14,15)}$.

Hallamos que dos tesis $(2,7 \%)$ de pregrado de la FMH-USMP fueron publicadas en alguna revista científica. En la UNMSM ${ }^{(7)}$ se ha descrito que $11,8 \%$ de las tesis de pregrado del periodo 1998-2008 fueron publicadas (en SciELO, Google Académico y LILACS). En la UPCH ${ }^{(8)}$, el $17,6 \%$ de las tesis de pregrado del periodo 2000-2003 fueron publicadas (en PubMed, Google Académico, LILACS, LIPECS y SciELO). Dichos estudios, al igual que el nuestro, han hecho uso de Google Académico, 
que busca teóricamente en todas las revistas científicas que tienen formato virtual. Queda claro que la UNMSM y la UPCH tienen tasas de publicación bastante más elevadas que la USMP. Para comprender mejor estos datos, es necesario mencionar que la UNMSM y la UPCH son las facultades de medicina con mayor producción científica en el Perú, tanto a nivel de profesionales ${ }^{(16,17)}$ como de estudiantes ${ }^{(3,18,19)}$.

En otros países, la publicación de tesis de pregrado sigue patrones variables. Por ejemplo, un estudio realizado en 36 universidades de Francia encontró que $17 \%$ de las tesis de pregrado del periodo 1993-1998 fueron publicadas en Medline ${ }^{(20)}$. También, se ha descrito la publicación de $23,8 \%$ de las tesis de pregrado realizadas en el periodo 2001-2003 en la universidad de Oulu (Finlandia) (en Medline, Scopus y otras bases de datos médicas) ${ }^{(21)}$. En la universidad de Lome (Francia), 41\% de las tesis de pregrado del periodo 1993-2002 fueron publicadas (en Medline y CNRS/PASCAL) ${ }^{(22)}$.

Otros artículos han analizado la publicación de las tesis de posgrado. Un estudio encontró que en una universidad de la India se publicó en Medline el 30\% de las tesis de maestría en medicina de los años 2001 al $2005^{(23)}$. Otro estudio en escuelas de medicina de Croacia mostró que $13,7 \%$ de tesis de maestría y $34,4 \%$ de tesis de doctorado fueron publicadas en Medline entre los años 1990 y $1999^{(24)}$. Estos estudios no pueden ser comparados con el nuestro, puesto que fueron realizados en otras bases de datos.

Para el desarrollo y la aprobación de las tesis de pregrado, debe existir una adecuada interacción entre los estudiantes, los asesores y los jurados, de tal manera que todos ellos contribuyan a elevar la calidad de las tesis y orienten al tesista hacia la publicación. Sin embargo, en nuestro estudio se halló que menos de la tercera parte de los asesores y jurados han publicado algún artículo original. Y los asesores de las dos tesis publicadas ya habían publi- cado artículos originales. Entonces, es posible que la inexperiencia de asesores y jurados esté relacionada con la baja publicación de las tesis, por lo cual es necesario que las facultades evalúen la producción científica de los asesores y jurados de las tesis de pregrado.

Resulta importante, también, que las facultades adecúen el formato de las tesis al formato requerido por las revistas científicas. Esto evitaría que el estudiante realice doble labor al redactar su trabajo de investigación como una tesis, y luego volver a escribirlo en el formato requerido por la revista científica, las cuales por lo general no aceptan más de 3500 palabras. Por ejemplo, las normas de la UPCH mencionan que los trabajos de investigación tendrán como máximo 14 páginas (sin incluir tablas, gráficos ni figuras), y es necesario que hayan sido enviados a alguna revista científica antes de ser aprobados ${ }^{(25)}$.

Se halló que la mayor parte de las tesis fueron realizadas en hospitales o institutos de Lima, posiblemente debido a que los asesores laboran en dichos hospitales y tienen facilidades para realizar trabajos de investigación allí.

Se encontró que en 43 tesis $(58,1 \%)$ se afirma tienen un diseño de tipo retrospectivo y $24(32,4 \%)$ un diseño transversal. Los artículos descriptivos y trasversales son los más frecuentes en los artículos originales publicados en revistas biomédicas peruanas en el período de 2002 al $2009{ }^{(26)}$. Se entiende entonces que el diseño utilizado no es una barrera para la publicación de artículos científicos en revistas peruanas.

En cuanto a las limitaciones del estudio, cabe mencionar que la estrategia de búsqueda utilizada para hallar la producción científica de los asesores y jurados no considera el caso de homonimia.

Nuestro estudio alerta sobre la baja publicación de tesis y analiza la producción científica de los asesores y jurados en una universidad peruana, por lo cual consideramos que será de utilidad para que las autoridades de las facultades de medicina apliquen los cambios curriculares y administrativos correspondientes, en favor de la correcta enseñanza de la investigación en el pregrado, que debe estar enfocada a la publicación científica.

En conclusión, se encontró que de 2667 graduados en la FMH-USMP durante el periodo 2000-2009, 75 lo hicieron mediante una tesis. En total se halló 74 tesis, de las cuales 2 fueron publicadas en revistas médicas indizadas. Solo 31,3\% de los asesores y 32,9\% de los miembros de jurado tenían algún artículo original publicado. Se sugiere que en el futuro se realice un estudio más amplio sobre el tema de publicación de tesis en el Perú.

\section{REFERENCIAS BIBLIOGRÁFICAS:}

1. Reinders JJ, Kropmans TJ, Cohen-Schotanus J. Extracurricular research experience of medical students and their scientific output after graduation. Med Educ. 2005;39(2):237.

2. Sogi C, Perales A. El quehacer de los Investigadores de la facultad de Medicina de la Universidad Nacional Mayor de San Marcos. An Fac med. 2001;62(2):100-14.

3. Taype-Rondán A, Lajo-Aurazo Y, Gutiérrez-Brown R, Zamalloa-Masias N, Saldaña-Gonzales M. Aporte de las Sociedades Estudiantiles en la publicación cientifica en SciELO-Perú. Rev Peru Med Exp Salud Publica. 2011;28(4):691-2.

4. Góngora M. El Estado, la universidad peruana y el drama de la investigación científica. Escritura y Pensamiento. 2002;5(11):43-57.

5. Hames I. Peer Review and Manuscript Management in Scientific Journals. Oxford: Blackwell Publishing; 2007.

6. Vilchez C, Nakamura D. Usabilidad de un sistema de recuperación de información a texto completo: el caso del portal Cybertesis Perú. ACIMED. 2008;17(3).

7. Valle R, Salvador E. Análisis bibliométrico de las tesis de pregrado de la Facultad de Medicina de la Universidad Nacional Mayor de San Marcos. An Fac med. 2009;70(1):11-8.

8. Arriola-Quiroz I, Curioso W, Cruz-Encarnacion M, Gayoso O. Characteristics and publication patterns of theses from a Peruvian medical school. Health Information and Libraries Journal. 2010;27(2):14854.

9. Mayta-Tristán P, Mezones-Holguin E. Aclaración editorial. Rev Peru Med Exp Salud Publica. 2009;26(3):411-2.

10. Pereyra-Elias R, Ng-Sueng L, Toro-Polo L, Nizama A, Piscoya A, Mayta-Tristán P. Baja publicación de los trabajos presentados a los Congresos de la Sociedad de Gastroenterología del Perú 1998-2008. Rev gastroenterol Perú. 2011;31(2):124-32.

11. Falagas ME, Pitsouni EI, Malietzis GA, Pappas G Comparison of PubMed, Scopus, Web of Science, and Google Scholar: strengths and weaknesses. FASEB J. 2008;22:338-42. 
12. Valle $R$, Salvador $E$. La tesis de medicina humana: experiencia en una universidad pública de Lima. Rev Peru Med Exp Salud Publica. 2009;26(2):270.

13. Ramos-Rodriguez M, Sotomayor R. Realizar o no una tesis: razones de estudiantes de medicina de una universidad pública y factores asociados. Rev Peru Med Exp Salud Publica. 2008;25(3):322-4.

14. Gutiérrez C, Mayta P. Publicación desde el pregrado en Latinoamérica: Importancia, limitaciones y alternativas de solución. CIMEL. 2003;8(1):54-60.

15. Alarco JJ, Aguirre-Cuadros E, Aliaga-Chávez Y, Álvarez-Andrade E. Factores asociados a la realización de tesis en pregrado de Medicina en una universidad pública del Perú. CIMEL. 2010;15(2):66-70.

16. Huamani $C$, Mayta-Tristàn $P$. Producción científica peruana en medicina y redes de colaboración, análisis del Science Citation Index 2000-2009. Rev Peru Med Exp Salud Publica. 2010;27(3):315-25.

17. Scimago research group. Ranking Iberoamericano SIR 2010. [En línea] [Citado 12 nov 2011]. Disponible en: http://www.scimagoir.com/pdf/ ranking_iberoamericano_2010.pdf.

18. Huamani C, Chávez-Solis P, Mayta-Tristán P. Aporte estudiantil en la publicación de artículos científicos en revistas médicas indizadas en Scielo-Perú 1997-2005. An Fac med. 2008;69(1):42-5.
19. Alarcón-Villaverde J, Romani F, Gutiérrez C. Publicaciones científicas estudiantiles producidas en el curso de Epidemiología de la Facultad de Medicina de la Universidad Nacional Mayor de San Marcos durante el periodo 2003-2009. An Fac med. 2010;71(2):111-6

20. Salmi LR, Gana S, Mouillet E. Publication pattern of medical theses, France, 1993-98. Med Educ. 2001;35(1):18-21.

21. Nieminen P, Sipilä K, Takkinen HM, Renko M, Ristel $L$. Medical theses as part of the scientific training in basic medical and dental education: experiences from Finland. BMC Med Educ. 2007;7:51.

22. Pitche PT, Onipoh D, Tchangai-Walla KL. Devenir scientifique des thèses pour le diplôme d'état de doctorat en médecine soutenues à l'université de Lomé (Togo). Pédagogie médicale. 2007;1:24-9.

23. Dhaliwal U, Singh N, Bhatia A. Masters theses from a university medical college: Publication in indexed scientific journals. Indian J Ophthalmol. 2010;58(2):101-4.

24. Frković V, Skender T, Dojćinović B, Bilić-Zulle L. Publishing Scientific Papers Based on Master's and Ph.D. Theses from a Small Scientific. Croat Med J. 2003;44(1):107-11.
25. Universidad Peruana Cayetano Heredia - Facultad de Medicina [sede Web]. Lima: UPCH FAMED; 2011 [acceso el 13 de mayo de 2012]. Normas y procedimientos para la elaboración, desarrollo, presentación, evaluación y publicación del trabajo de investigación. Disponible en: http://www.upch.edu. pe/famed/alumno/pregrado/medicina/investigacion/ titulo.asp

26. Romani F, Márquez J, Wong P. Uso de los métodos estadísticos en artículos originales de cinco revistas biomédicas peruanas. Periodo 2002-2009. Rev per epidemiol. 2010;14(2):153-60.

Artículo recibido el 2 de marzo de 2012 y aceptado para publicación el 22 de abril de 2012.

\section{Correspondencia:}

Álvaro Taype-Rondán

Dirección: Calle 22 N¹59, Dpto. 102, Urb Corpac,

San Borja, Lima, Perú.

Teléfonos: 998929945 / 2265586

Correo electrónico: alvaro_athos@hotmail.com 